\title{
Imaging biomarkers in the diagnosis of salivary gland tumors: the value of lesion/parenchyma ratio of perfusion-MR pharmacokinetic parameters
}

\author{
Francesco Mungai ${ }^{1}\left({ }^{10} \cdot\right.$ Giovanni Battista Verrone $^{1} \cdot$ Luigi Bonasera $^{1} \cdot$ Eleonora Bicci $^{1} \cdot$ Michele Pietragalla $^{2}$. \\ Cosimo Nardi ${ }^{2} \cdot$ Valentina Berti $^{2} \cdot$ Lorenzo Nicola Mazzoni $^{3} \cdot$ Vittorio Miele $^{1}$
}

Received: 12 November 2020 / Accepted: 12 May 2021

(c) Italian Society of Medical Radiology 2021

\begin{abstract}
Background and purpose Morphologic magnetic resonance imaging (MRI) for characterization of salivary gland tumors has limited utility, and the use of perfusion MRI data in the clinical setting is controversial. We examined the potential of tissue-normalized dynamic contrast-enhanced (DCE) MRI pharmacokinetic parameters of salivary gland tumors as imaging biomarkers for characterization and differentiation between benign and malignant lesions.

Materials and methods DCE-MR images acquired from 60 patients with parotid and submandibular gland tumors were retrospectively reviewed. Pharmacokinetic parameters as transfer constant (Ktrans), rate constant (Kep), extracellular space volume ( $V e$ ), fractional plasma volume ( $V p)$, and $A E C$ (area of all times enhancement curve) were measured on both the lesion and the normal contralateral salivary gland parenchyma. Lesion/parenchyma ratio (L/P) for each parameter was calculated. Results Five groups of lesions were identified (reference: histopathology): pleomorphic adenomas $(n=20)$, Warthin tumors $(n=16)$, other benign entities $(n=4)$, non-Hodgkin lymphomas $(n=4)$, and malignancies $(n=16)$. Significant differences were seen for mean values of L/PKtrans (higher in malignancies), L/PKep (lower in adenomas than Warthin tumors), $\mathrm{L} / \mathrm{PVe}$ (lower in Warthin tumors and lymphomas), L/PVp (higher in Warthin tumors and malignancies than adenomas), and $\mathrm{L} / \mathrm{PAEC}$ (higher in malignancies). Significant differences were found between benign and malignant (non-lymphoproliferative) lesions in mean value of L/PKtrans (0.485 and 1.581), L/PVp (1.288 and 2.834), and L/PAEC (0.682 and 1.910). ROC analysis demonstrated the highest AUC (0.96) for L/PAEC, with sensitivity and specificity for malignancy of $93.8 \%$ and $97.5 \%$ (cutoff value $=1.038$ ).

Conclusion Lesion/parenchyma ratio of DCE-MRI pharmacokinetic data could be helpful for recognizing the principal types of salivary gland tumors; L/PAEC seems a valuable biomarker for differentiating benign from malignant tumors.
\end{abstract}

Keywords Salivary gland tumors · Dynamic contrast-enhanced MRI $\cdot$ Pharmacokinetic analysis · Imaging biomarkers · Lesion/parenchyma ratio

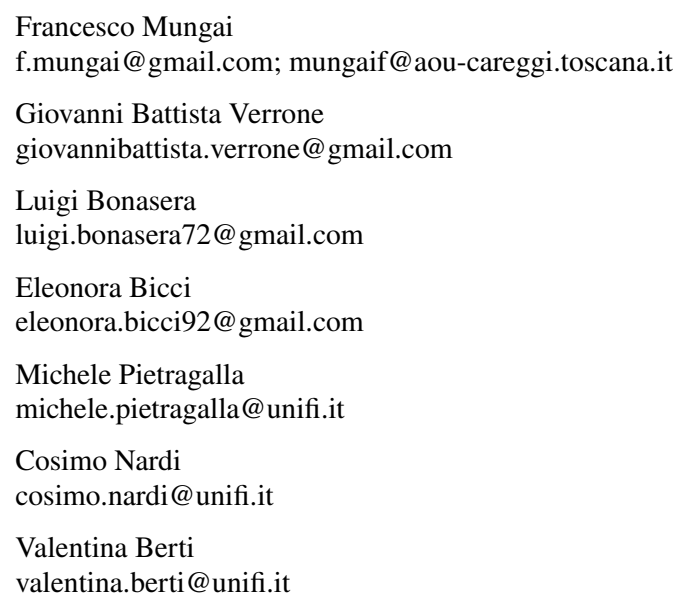

Lorenzo Nicola Mazzoni mazzoniln@gmail.com

Vittorio Miele

vittoriomiele@gmail.com

1 Department of Diagnostic Imaging, Careggi University Hospital, Largo Brambilla 3, 50134 Firenze (FI), Italy

2 Department of Experimental and Clinical Biomedical Sciences, School of Human Health Sciences, University of Florence, Largo Brambilla 3, 50134 Firenze (FI), Italy

3 Department of Medical Physics, Careggi University Hospital, Largo Brambilla 3, 50134 Firenze (FI), Italy 


\section{Introduction}

In patients with salivary gland tumors, the distinction between benign and malignant neoplasm is critical, since the latter demands more aggressive intervention [1]. Magnetic resonance imaging (MRI) represents the primary preoperative diagnostic modality in patients with salivary gland tumors. Routine pre- and post-contrast MR sequences allow determining the extent of disease (localization of the primary tumor and regional lymph nodes involvement) and evaluating morphologic features of the lesion such as dimensions, shape, margins, and the presence of cystic/necrosis/fluid areas [2]. Still, various benign and malignant tumors often show non-specific imaging findings and overlapping morphologic characteristics [3-6], so differentiation between the two groups is generally tricky.

Current clinical guidelines advise obtaining a lesion sample by the use of fine-needle aspiration cytology (FNAC) or ultrasound-guided core needle biopsy (CNB) [1]. However, FNAC presents limited sensitivity and a high number of non-diagnostic samples [7] while CNB, despite the diagnostic superiority [8], is still not considered the reference standard due to invasiveness, possible false negatives, and risk of tumor seeding [9]. For these reasons, many efforts are recently put into determining if multiparametric and advanced MR sequences such as diffusion-weighted imaging (DwI) and dynamic contrast-enhanced (DCE) imaging could have a role in characterizing salivary gland tumors [10-13] and differentiating benign from malignant lesions [12-15], with various results.

DCE-MRI provides biomarkers of tissue perfusion with proven utility in oncologic imaging and has been widely employed for diagnosis, prognosis, and therapy monitoring of cancer. Nevertheless, obtaining reproducible and clinically feasible imaging biomarkers from perfusion and permeability analysis is still a challenge because conspicuous repeatability and reproducibility data are lacking. Sources of variables in quantitative DCE-MRI analysis are various [16], and measured perfusion parameters broadly vary across different research sites, preventing data comparison in multiinstitutional clinical trials. As a result, in routine clinical practice, DCE-MRI is prevalently analyzed only qualitatively, with possible operator-dependent different results. To overcome these problems, it could be useful having a specific per-patient reference parameter such as the standard reference tissue. The ratio of DCE-MR pharmacokinetic parameters between a lesion and the standard reference tissue could potentially mitigate the variability linked to external factors and provide a more reliable representation of the intrinsic microvascularity and interstitial changes occurring in the different lesions with respect to the normal tissue [17].
Therefore, the purpose of this study was to examine the potential of tissue-normalized DCE-MRI pharmacokinetic parameters of salivary gland tumors as imaging biomarkers for characterization and differentiation between benign and malignant lesions.

\section{Materials and methods}

\section{Patients}

Our institutional review board approved this retrospective study (protocol number 2042/2019). Informed consent was obtained from all patients. We reviewed MR imaging performed from January 2015 to November 2018 in 72 consecutive patients with known (from clinical examination and previous ultrasound/CT imaging) parotid or submandibular gland tumors. All of these tumors were characterized both by histology analysis derived from ultrasound-guided CNB and histopathological confirmation when surgically excised. The other inclusion criteria of this study were: the maximum diameter of the lesion greater than $1 \mathrm{~cm}$ with the presence of enough non-cystic areas (at least 50\% of the entire volume of the lesion); the availability of DCE-MRI images without significant susceptibility or motion artifacts; patients had no previous radiation treatment on head and neck district nor known chronic inflammatory salivary gland disease (i.e., Sjogren's disease). Consequently, we excluded eight patients due to low image quality related to motion artifacts and two patients because of the predominant cystic component of the tumors. Two patients with bilateral tumors were also excluded from the study.

Resulting 60 lesions were then categorized into five different groups based on histopathology results: pleomorphic adenoma, Warthin tumors, other benign entities, non-Hodgkin lymphoma, and malignancies.

\section{Conventional and DCE-MR imaging acquisitions}

All MRI studies were performed by using a 1.5-T MR scanner (MAGNETOM Aera, Siemens Healthcare, Erlangen, Germany) with a dedicated head coil. Our protocol (Table 1) included morphologic imaging constituted by 3D-T1w and 3D-FS T2w sequences, axial TSE T2w imaging, and DwI. For DCE acquisition, we used a 3D fast spoiled gradient recalled echo $\mathrm{T} 1 \mathrm{w}$ imaging obtained after intravenous administration of $0.1 \mathrm{mmol} / \mathrm{kg}$ bodyweight of Gadobutrol (Gadovist, Bayer Schering Pharma AG) at an injection rate of $3 \mathrm{ml} / \mathrm{s}$, followed by a 20-ml saline flush. After contrast injection, at least 50 phases were acquired, covering more than 4 min with a temporal resolution of $5 \mathrm{~s}$. DCE acquisitions were preceded by a variable flip angle (FA $5^{\circ}$ and $15^{\circ}$ ) series, for pre-contrast agent native tissue $\mathrm{T} 1$ mapping. 
Table 1 Imaging Protocol

\begin{tabular}{lllllll}
\hline Parameters & SPACE T1w & SPACE T2w FS & TSE T2w & DWI EPI SPAIR & VIBE Dixon T1w & DCE \\
\hline TR (ms) & 500 & 3000 & 5050 & 4400 & 10 & 4.65 \\
TE (ms) & 7.2 & 380 & 117 & 54 & TE 1: 2.39 TE 2: 4.47 & 1.66 \\
FA (degree) & VFA & VFA & $90^{\circ}-150^{\circ}$ & $90^{\circ}$ & $10^{\circ}$ & $30^{\circ}$ (two preliminary \\
sequences with $5^{\circ}$ and \\
\end{tabular}

$T R$ Time of repetition; $T E$ time of echo; $F A$ flip angle; VFA variable flip angle; $F O V$ field of view; $S T$ slice thickness; $T A$ time of acquisition; GRAPPA generalized autocalibrating partially parallel acquisition; SPACE sampling perfection with application optimized contrasts using different flip angle evolution; FS fat saturation; DWI diffusion-weighted imaging; EPI echo planar imaging ; SPAIR spectral selection attenuated inversion recovery; VIBE volumetric interpolated breath hold examination; $D C E$ dynamic contrast enhanced

\section{Post-processing of DCE-MRI images}

Post-processing of acquired DCE-MR images was performed by using the application MR Permeability available inside the commercial software IntelliSpace Portal v.8.0 (Philips Healthcare, Best, The Netherlands). DCEMRI data were elaborated in distinctive steps, according to what has been recommended by QIBA (Quantitative Imaging Biomarker Alliance) initiative [18]:

- Generating a native tissue T1 map using the variable flip angle data

- Applying time-series motion correction to the dynamic data

- Converting DCE-MRI signal intensity data, SI(t), to gadolinium concentration $([\mathrm{Gd}](\mathrm{t}))$

- Calculating a vascular input function (VIF)

The pharmacokinetic calculation was done on a pixelby-pixel basis using a 2-compartment model, based on the extended Tofts model (ETM) [19]:

$\mathrm{C}(\mathrm{t})=V p * \mathrm{Ca}(t)+K$ trans $* \mathrm{e}^{-\mathrm{t} K e p} * \mathrm{Ca}(t)$

where:

$\mathrm{C}(\mathrm{t})$ : Contrast concentration in tissue.

$V p$ : The fractional value of blood plasma (also referred to as volume fraction of plasma space).

$\mathrm{Ca}(t)$ : VIF (contrast concentration in feeding artery).
Ktrans: Transfer constant between blood plasma and extravascular extracellular blood space (EES).

Kep: Rate constant between EES and blood plasma (Ktrans/Ve).

We chose to apply a model-based VIF with a fixed biexponential shape related to the type and dose of injected contrast media $(0.1 \mathrm{mmol} / \mathrm{kg}$ of Gadobutrol), the duration of contrast injection (5 to $10 \mathrm{~s}$ ), and an average hematocrit value of $45 \%$. Then, we carried out a pixel-by-pixel analysis to generate color-coded maps of Ktrans, Kep, Ve (fractional EES volume), $V p$, and $A E C$ (area of all times enhancement curve) for the whole volume.

\section{Regions of interest (ROI) selection and extraction of DCE parameters}

Morphologic and DCE-MR images were reviewed in consensus by two radiologists (F.M. and G.V) with 12 and five years of experience in head and neck imaging, blinded to the histopathology results. An ROI encompassing as much of the tumor area as possible was manually drawn, excluding large cystic or necrotic areas. Then, preferably on the same slice of the acquisition volume, another ROI with comparable area size was drawn inside the contralateral visually normal salivary gland parenchyma, excluding large vessels, such as the retromandibular vein, and normal intraparenchymal lymph nodes. 
Since the lesion and the normal salivary gland parenchyma were well delineated on contrast-enhanced $\mathrm{T} 1 \mathrm{w}$ images, we used the dynamic series of DCE acquisition for ROIs drawing. We created summation images (obtained by adding together images acquired on the dynamic series for each slice location), and we used average images to perform the segmentation (Fig. 1a). As these segmentations were registered in the dynamic set, we used them to directly extract lesion- and parenchyma-based parameters from parametric maps. Visual analysis of the time-signal intensity curve (TIC) resulting from each segmentation was carried out for quality assessment (Fig. 1a); when a non-viable curve occurred, the segmentation was repeated. We also performed a visual assessment of the generated color-coded maps of Ktrans, Kep, Ve, Vp, and AEC (Fig. 1b).

We finally obtained the lesion/parenchyma ratio $(\mathrm{L} / \mathrm{P})$ of the DCE parameters (L/P Ktrans, L/P Kep, L/P Ve, L/P Vp, and $\mathrm{L} / \mathrm{P} A E C$ ) by dividing the mean values measured in the two ROIs for each patient.

\section{Statistical analysis}

Because of the small sample size of the five groups and the non-normal distribution of the data, the Kruskal-Wallis $\mathrm{H}$ test was performed to assess whether there were any statistically significant differences between the means of the five different groups of lesions in the calculated parameters (L/P Ktrans, L/P Kep, L/P Ve, L/P Vp, L/P AEC). Dunn's pairwise test (using the Bonferroni correction) was then run to confirm the differences that occurred between the groups.

Then, $t$ test and receiver operator characteristics (ROC) analysis were performed to find out the performance of the lesion/parenchyma ratio of DCE parameters for the distinction between the benign lesions (including pleomorphic adenoma, Warthin tumors, and the other benign entities) and the malignancies (excluding non-Hodgkin lymphomas). We finally performed the discriminant analysis for assessing the performance of the lesion/parenchyma ratio of DCE parameters in isolation or in combination for discriminating between benign and malignant lesions. All statistical analyses were performed by using SPSS statistic software (v.25; IBM SPSS, Chicago, Ill.).

\section{Results}

Resulting 60 lesions were then categorized into five different groups based on histopathology results: pleomorphic adenoma $(n=20)$, Warthin tumors $(n=16)$, other benign entities $(n=4)$, non-Hodgkin lymphoma $(n=4)$, and malignancies $(n=16)$. Of 60 patients, there were finally 40 benign tumors, 16 malignancies, and 4 non-Hodgkin lymphomas. Origin, maximum diameter, and histopathological diagnosis of the tumors are shown in Table 2. Descriptive analysis for L/P Ktrans, L/P Kep, L/P Ve, L/P Vp, and L/P AEC in the five groups is shown in Table 3. Kruskal-Wallis and Dunn's pairwise tests showed the following statistically significant differences among groups. The group of malignancies presented higher mean L/P Ktrans value than the groups of pleomorphic adenomas $(1.581$ versus $0.440, P<0.001)$, Warthin tumors $(0.510, P=0.001)$, and lymphomas $(0.366$, $P=0.006)$. The group of pleomorphic adenomas presented lower mean L/P Kep values than the groups of Warthin tumors $(0.323$ vs. $0.861, P<0.001)$, and malignancies (0.659, $P=0.05)$. Lower mean $\mathrm{L} / \mathrm{P} V e$ values were found in Warthin tumors respect to pleomorphic adenomas $(0.652$ vs. $1.868, P=0.001)$ and malignancies $(2.524, P<0.001)$; lymphomas also had lower mean L/P Ve than malignancies $(0.479$ vs. $2.524, P=0.001)$ and pleomorphic adenomas $(P=0.025)$. Differences in mean L/P $V p$ were found when comparing pleomorphic adenomas to Warthin tumors (0.418 vs. $2.206, P<0.001)$ and malignancies $(2.834$, $P<0.001)$; lymphomas also had lower mean L/P $V p$ than Warthin tumors $(0.317$ vs. $2.206, P=0.012)$ and malignancies $(P=0.025)$. Finally, mean $\mathrm{L} / \mathrm{P} A E C$ values were higher in malignancies respect to pleomorphic adenomas (1.910 vs $0,584, P<0.001)$, Warthin tumors $(0.745, P<0.001)$, and lymphomas $(0.342, P=0.002)$. In Table 4 , a summary of types of lesions with L/P ratio higher or lower than 1.0 is described.

After excluding the $n=4$ lymphomas from the analysis, we found statistically significant differences between benign and malignant lesions in mean value of L/P Ktrans (respectively, 0.485 and $1.581, P=0.001), \mathrm{L} / \mathrm{P} V p(1.288$ and $2.834, \mathrm{P}=0.006)$, and $\mathrm{L} / \mathrm{P} A E C(0.682$ and $1.910, P<0.001)$ (Table 5 and Fig. 2). ROC analysis demonstrated an area under the curve, respectively, of 0.933 for L/P Ktrans and 0.955 for L/P AEC (Fig. 3). By applying the cutoff L/P AEC value of 1.038 , sensitivity and specificity in discriminating malignant from benign lesions were, respectively, 93.8\% and $97.5 \%$. Finally, discriminant analysis with a stepwise approach demonstrated that the $\mathrm{L} / \mathrm{P} A E C$ alone is better than any other combination of parametric ratios in differentiating malignancy from a benign lesion (Figs. 4, 5).

\section{Discussion}

This study investigated the potential use of the lesion/parenchyma ratio of DCE-MRI pharmacokinetic parameters for characterization and differentiation between benign and malignant salivary gland tumors. DCE-MRI, especially when acquired using a high temporal resolution $(<6 \mathrm{~s}$ per volume acquisition), is a useful technique to noninvasively disclose the changes in microvascular perfusion and extracellular space volume of tumors with respect to standard 
Fig. 1 57-year-old woman with Warthin tumor of the left parotid gland (white arrow). ROI selection and extraction of DCE-MRI parametric maps. Figure a demonstrates the delineation of the lesion (ROI 1-blue line), and the normal contralateral parotid parenchyma (ROI 2-orange line) on the dynamic series of DCE acquisition; corresponding time-signal intensity (TIC) curves of the two ROIs are shown below. Matching color-coded maps of Ktrans, $V e, V p$, and $A E C$ are displayed in Fig. b. Lesion/ parenchyma ratio $(\mathrm{L} / \mathrm{P})$ values found for this case were: $\mathrm{L} / \mathrm{P}$ Ktrans $=0.59, \mathrm{~L} / \mathrm{P}$ Kep $=1.05$, $\mathrm{L} / \mathrm{P} V e=0.57, \mathrm{~L} / \mathrm{P} V p=3.42$, and $\mathrm{L} / \mathrm{P} A E C=0.83$
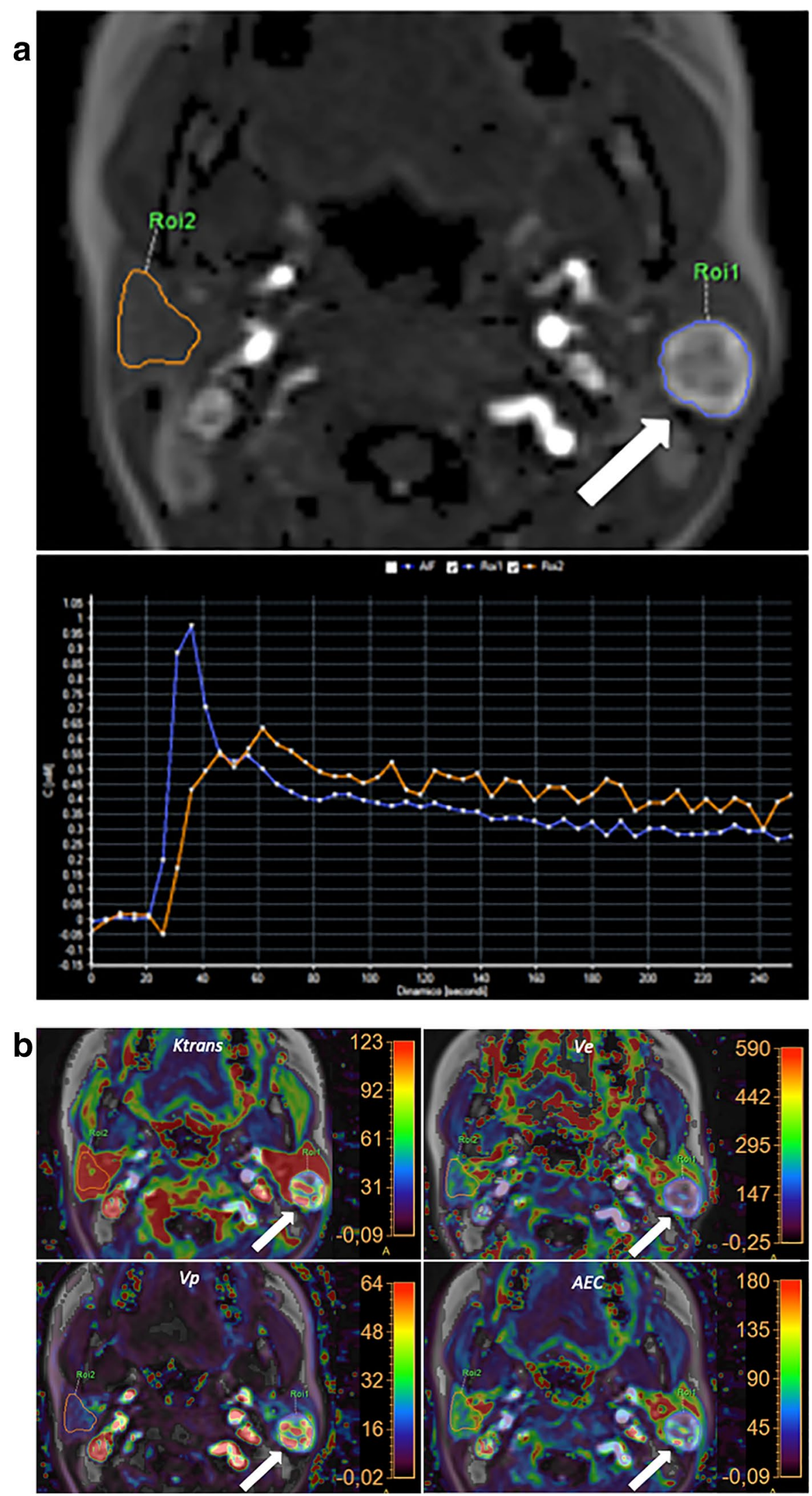
Table 2 Summary of salivary lesions and patients characteristics

\begin{tabular}{lllll}
\hline Histology (number of lesions) & Site (number of lesions) & Patient sex & Patient age (range) & Diameter in cm (range) \\
\hline Pleomorphic adenoma (20) & Parotid (17) Submandibular (3) & 13 female; 7 male & $47.8(27-76)$ & $2.6(1-4.7)$ \\
Warthin tumor (16) & Parotid (16) & 5 female; 11 male & $64(54-71)$ & $1.8(1.5-2.5)$ \\
Other benign tumors (total =4): & Parotid (4) & 2 female; 2 male & $72.2(68-80)$ & $2.4(1.4-3.5)$ \\
Myoepithelioma (2) & Parotid (1) & 1 female; 1 male & $75(70-80)$ & $2.85(2.2-3.5)$ \\
Oncocytoma (1) & Parotid (1) & 1 female & 71 & 1.4 \\
Basal cell adenoma (1) & Parotid (1) & 1 male & 68 & 2.4 \\
Non-Hodgkin lymphoma (4) & Parotid (4) & 2 female; 2 male & $65(60-68)$ & $2.9(2.2-3.6)$ \\
Malignant lesions (total=16): & Parotid (13) Submandibular (4) & 9 female; 7 male & $60.5(39-75)$ & $2.7(1.2-6)$ \\
Cystic adenoid carcinoma (3) & Parotid (1) Submandibular (2) & 2 female; 1 male & $65.7(63-70)$ & $2.5(2-2.8)$ \\
Mucoepidermoid carcinoma (3) & Parotid (1) Submandibular (2) & 1 female; 2 male & $53.7(50-59)$ & $1.9(1.2-2.5)$ \\
Carcinoma ex pl. adenoma (3) & Parotid (3) & 3 female & $49.3(39-60)$ & $2.5(1.3-3.1)$ \\
Salivary duct adenocarcinoma (1) & Parotid (1) & 1 male & 71 & 2.9 \\
Basal cell adenocarcinoma (1) & Parotid & 1 female & 70 & 1.7 \\
Squamous cell carcinoma (2) & Parotid (2) & 1 female; 1 male & $72(69-75)$ & $2.5(2-3)$ \\
Undifferentiated carcinoma (2) & Parotid (2) & 2 male & 58 & $3.7(2.9-4.5)$ \\
Clear cell carcinoma metastasis (1) & Parotid & 1 female & 60 & 6 \\
\hline
\end{tabular}

Table 3 Descriptive analysis for L/P Ktrans, L/P Kep, L/P Ve, L/P Vp, and L/P AEC in the five main groups of lesions

\begin{tabular}{llllll}
\hline Histology & L/P Ktrans & L/P $K e p$ & L/P $V e$ & L/P $V p$ & L/P $A E C$ \\
\hline Pleomorphic adenoma $(n=20)$ & $0.440 \pm 0.249^{*}$ & $\mathbf{0 . 3 2 3} \pm \mathbf{0 . 2 5 2} *$ & $\mathbf{1 . 8 6 8} \pm \mathbf{1 . 3 8 4} *$ & $0.418 \pm 0.458^{*}, * *$ & $0.584 \pm 0.256^{*}$ \\
Warthin tumor $(n=16)$ & $0.511 \pm 0.970^{*}$ & $0.861 \pm 0.247^{*}$ & $0.652 \pm 0.218^{*}$ & $\mathbf{2 . 2 0 6} \pm \mathbf{0 . 9 9 8} *^{*}$ & $0.745 \pm 0.127^{*}$ \\
Other benign entities $(n=4)$ & $0.611 \pm 0.211$ & $0.809 \pm 0.448^{*}$ & $0.898 \pm 0.373$ & $2.061 \pm 2.138$ & $0.919 \pm 0.318$ \\
Non-Hodgkin lymphoma $(n=4)$ & $0.366 \pm 0.188^{*}$ & $0.621 \pm 0.101$ & $0.479 \pm 0.223^{* *}$ & $0.317 \pm 0.292$ & $0.342 \pm 0.249^{*}$ \\
Malignant lesions $(n=16)$ & $\mathbf{1 . 5 8 1} \pm \mathbf{1 . 3 9 3} *$ & $0.658 \pm 0.411^{*}$ & $\mathbf{2 . 5 2 4} \pm \mathbf{1 . 2 7 8}^{* *}$ & $\mathbf{2 . 8 3 4} \pm \mathbf{2 . 8 1 6} *^{*}$ & $\mathbf{1 . 9 1 0}^{*} \mathbf{1 . 2 2 6}^{*}$
\end{tabular}

Data are mean values \pm standard deviation

* and ** refer to correlated significant different $(<0.05)$ values for each parameter (in bold the highest or the lowest)

Ktrans transfer constant between blood plasma and extravascular extracellular blood space (EES); Kep rate constant between EES and blood plasma (Ktrans/Ve); Ve fractional EES volume; $V p$ fractional plasma volume; $A E C$ area of all times enhancement curve

Table 4 Summary of groups of lesions with mean L/P greater or lower than 1.0

\begin{tabular}{llllll}
\hline Histology & L/P Ktrans & L/P Kep & L/P Ve & L/P Vp & L/P AEC \\
\hline Pleomorphic adenoma $(n=20)$ & $<1$ & $<1$ & $>1$ & $<1$ & $<1$ \\
Warthin tumor $(n=16)$ & $<1$ & $<1$ & $<1$ & $>1$ & $<1$ \\
Other benign entities $(n=4)$ & $<1$ & $<1$ & $<1$ & $>1$ & $<1$ \\
Non-Hodgkin lymphoma $(n=4)$ & $<1$ & $<1$ & $<1$ & $<1$ & $<1$ \\
Malignant lesions $(n=16)$ & $>1$ & $<1$ & $>1$ & $>1$ & $>1$ \\
\hline
\end{tabular}

$L / P$ Lesion/parenchyma ratio; Ktrans transfer constant between blood plasma and extravascular extracellular blood space (EES); Kep rate constant between EES and blood plasma (Ktrans/Ve); Ve fractional EES volume; $V p$ fractional plasma volume; $A E C$ area of all times enhancement curve origin tissue [20]. Parotid, submandibular, and other salivary glands are constituted by a normally variegated tissue, mainly composed of serous and mucinous acini, an oncocytic epithelium forming intercalated and striated ducts and surrounded by myoepithelial cells, some sebaceous elements, adipose cells (more abundant in the parotids with a ratio of adipose to glandular tissue up to 1:1), and higher microvascular density than adjacent soft tissues; inside the parotids there are also about ten lymph nodes [21]. As a result, normal salivary gland parenchyma usually presents prominent vascular perfusion with a TIC curve composed by an early peak of contrast enhancement (40 to $60 \mathrm{~s}$ after 
Table 5 Mean values of DCE parameters in benign and malignant lesions with $\mathrm{t}$ test significance

\begin{tabular}{llll}
\hline DCE parameters & Benign lesions $(n=40)$ & $\begin{array}{l}\text { Malignant } \\
\text { lesions } \\
(n=16)\end{array}$ & $p$ value \\
\hline L/P Ktrans & $0.485 \pm 0.201$ & $1.581 \pm 1.393$ & $0.001^{*}$ \\
L/P Kep & $0.587 \pm 0.376$ & $0.658 \pm 0.411$ & 0.443 \\
L/P Ve & $1.285 \pm 1.147$ & $2.524 \pm 1.280$ & 0.309 \\
L/P Vp & $1.297 \pm 1.277$ & $2.834 \pm 2.816$ & $0.006^{*}$ \\
L/P $A E C$ & $0.682 \pm 0.241$ & $1.910 \pm 1.226$ & $0.0001^{*}$ \\
\hline
\end{tabular}

Except where otherwise indicated, data are mean values \pm standard deviation

*Significant difference $(<0.05)$

intravenous injection) followed by a slow washout. This perfusion curve reflects on quantitative DCE parameters, resulting in higher values of Ktrans, Kep, Vp, and AEC than, for example, adjacent soft tissues, when visually evaluated on correspondent color maps or measured by placing ROIs. In salivary glands, tumors cellularity (type and density), microarchitecture, and microcirculation are different from normal parenchyma, and our results demonstrated that these changes could be disclosed by DCE analysis. In our series, pleomorphic adenoma presented increased $V e$ and reduced $V p$ values than normal salivary parenchyma with mean L/P ratio values, respectively, of 1.868 and 0.418 ; this could be related to the presence of some myxoid stromal component inside this type of tumor and reflects the generally high values of apparent diffusion coefficient (ADC) derived by Dw images. On the other side, Warthin tumor due to its high density of cells (oncocytic epithelium and follicles containing lymphoid tissue) and microvessels presented decreased $V e$ (mean L/P ratio value 0.652 ) and increased $V p$ (2.206) than normal parenchyma. Lymphoproliferative lesions are usually composed of follicles containing very high cell density and a quite low vascularization, so reduced extracellular and vascular space volume brought to lower L/P Ve (0.479) and $V p(0.317)$, as expected. The low extracellular space volume of both Warthin tumor and lymphoma is also highlighted by the presence of restricted diffusion of both on Dw images, generally greater for lymphoma.

In our series clinically benign lesions including pleomorphic adenoma, Warthin tumors and other histotypes (such as myoepithelioma, basal cell adenoma, and oncocytoma) presented mean values of Ktrans ad AEC well lower than normal parenchyma (mean L/P ratio, respectively, of 0.485 and 0.682 ) and this was just clearly evident by visually analyzing the color maps, other than by measuring values by ROIs. Malignancies, as expected due to the presence of prominent neovascularization, in our series show very well increased values of Ktrans, $V p$, and $A E C$ than normal parenchyma

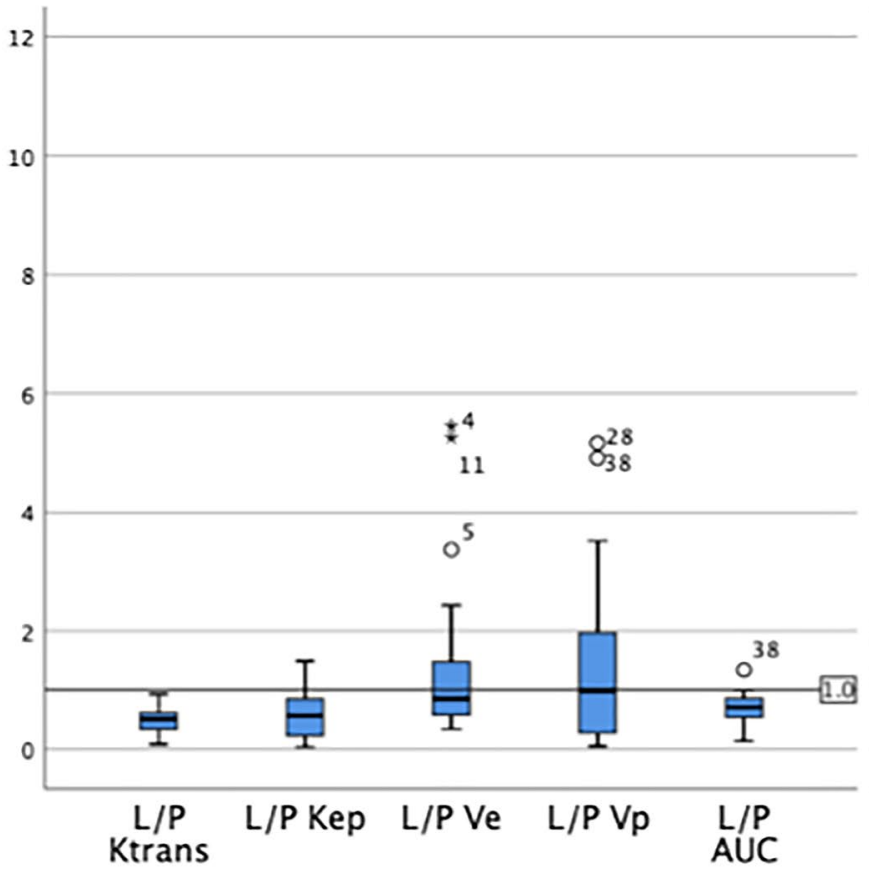

Fig. 2 Box-and-whisker plots chart representing values of lesion/ parenchyma ratio (L/P) of DCE-MRI pharmacokinetic parameters in benign (blue boxes) and malignant salivary gland tumors (red boxes, excluding the $n=4$ cases of non-Hodgkin lymphoma). The lower and upper hinges of the boxes denote the 25 th and 75 th percentiles,

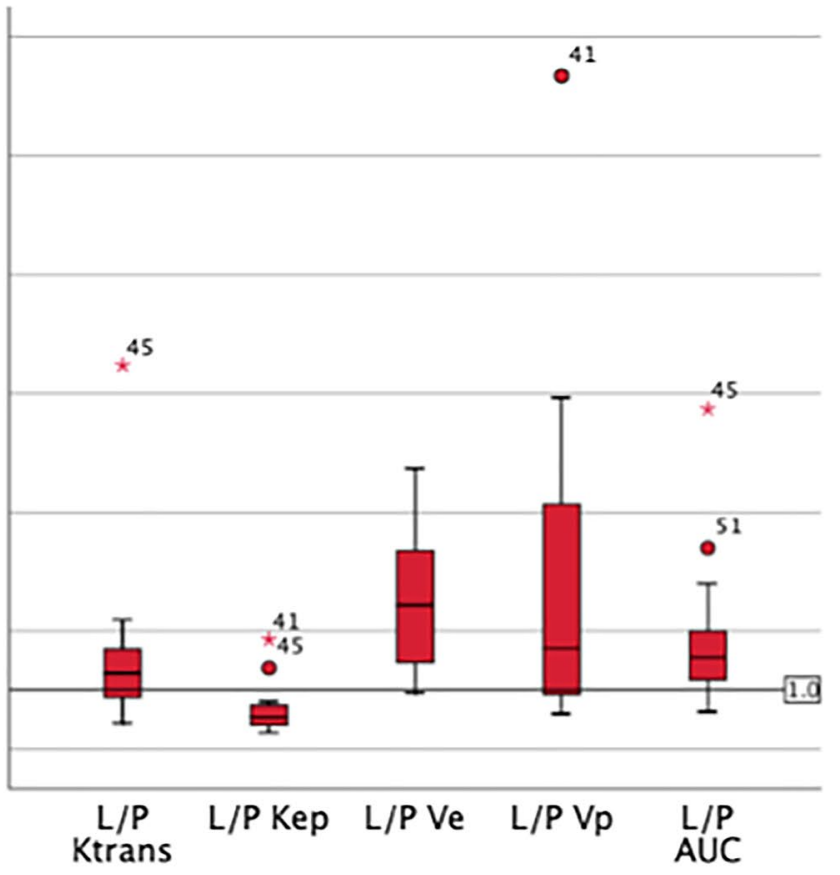

respectively. The median (50th percentile) of each distribution is indicated by the line. Malignant tumors presented ratio values mostly above the reference line of 1.0 with significant differences in mean values of L/P Ktrans $(p=0.001), \mathrm{L} / \mathrm{P} V p(p<0.01)$, and L/P AEC $(p<0.001)$ 
Fig. 3 ROC curves of lesion/ parenchyma ratio (L/P) of DCE-MRI pharmacokinetic parameters for the differentiation between malignant and benign salivary gland tumors. The area under the curve was, respectively, of 0.93 for $\mathrm{L} / \mathrm{P}$ Ktrans (blue line) and 0.96 for $\mathrm{L} / \mathrm{P} A E C$ (orange line). By applying the cutoff $\mathrm{L} / \mathrm{P} A E C$ value of 1.038 , sensitivity and specificity in discriminating malignant from benign lesions were, respectively, $93.8 \%$ and $97.5 \%$

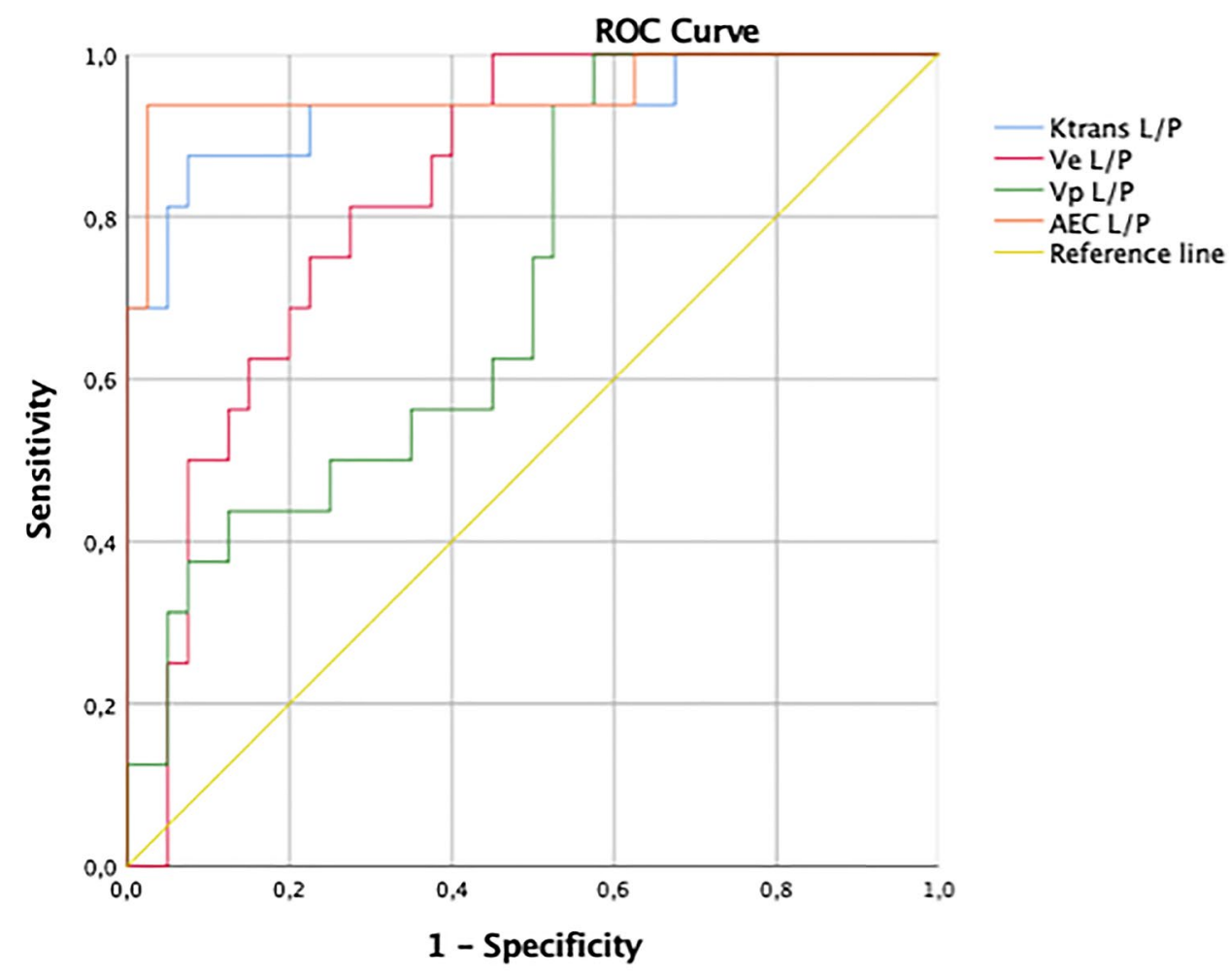

(corresponding mean L/P of 1.581, 2.834, and 1.910), and also this was just clearly visible on color maps. Tumor neoangiogenesis brings higher microvascular density in malignancies than normal reference tissue and benign tumors; this reflects higher $V p$. Moreover, increased microvascular permeability can be observed because of larger fenestrations in capillary vessels. Consequently, a greater amount of contrast medium can pass into extracellular interstitial space, reflecting higher Ktrans and AEC of malignant tumors [22]. Therefore, L/P of Ktrans and $A E C$ were advantageous in differentiating malignancies from clinically benign tumors, as demonstrated by ROC analysis. The cutoff value for $\mathrm{L} / \mathrm{P}$ $A E C$ with the highest values of sensitivity and specificity (respectively, of 93.8 and 97.5\%) was 1.038, and this appears significant in terms of clinical ease of use of the method. In fact, in our series by only examining the $A E C$ color map in the majority of cases we were quite easily able to visually recognize if the lesion had a color pattern representing higher or lower $A E C$ value than normal parenchyma and, consequently, if it appeared suspicious for malignancy, even without the need of drawing the two ROIs and calculating the ratio.

Our study presents the following limitations. First of all, it is a retrospective study with a relatively small cohort of patients and quantitatively non-homogenous histological groups, that, however, reflect the prevalence of the different salivary gland tumor histotypes in the population. Secondly, in the process of distinguishing malignant from benign tumors, some problems may arise when cystic or necrotic areas are present inside a tumor. We have found that if intratumoral cystic/necrotic areas comprised less than about half of the tumoral surface on a given slice, the perfusion curve of the inclusive ROI was still viable and quantitative parameters of the non-cystic part of the tumor were not significantly affected. On the contrary, when larger cystic/ necrotic areas were present, the measured perfusion curve of the inclusive ROI was generally unreliable, so we had to exclude them from the analysis, if possible, to get meaningful measurements of DCE parameters. In the end, when we faced an entirely cystic lesion, we acknowledged the perfusion would not be helpful, so the differential diagnosis should be derived by looking at conventional morphologic and DwI.

Moreover, during routinary clinical practice, it could be possible to face the presence of bilateral salivary gland involvement. This problem in our experience occurred only in the case of Warthin tumors, which usually are capsulated and have well-defined margins. In such a case, it could be hypothesized that the presence of the tumor itself should not alter the peritumoral salivary gland parenchyma, and reliable results can be obtained by correlating the DCE parametric values of the lesion to those obtained in the morphologically normal adjacent parenchyma. Lastly, older and obese patients may present dystrophic changes and more abundant fat infiltration of the parotid tissue that can alter normal microcirculation (with 

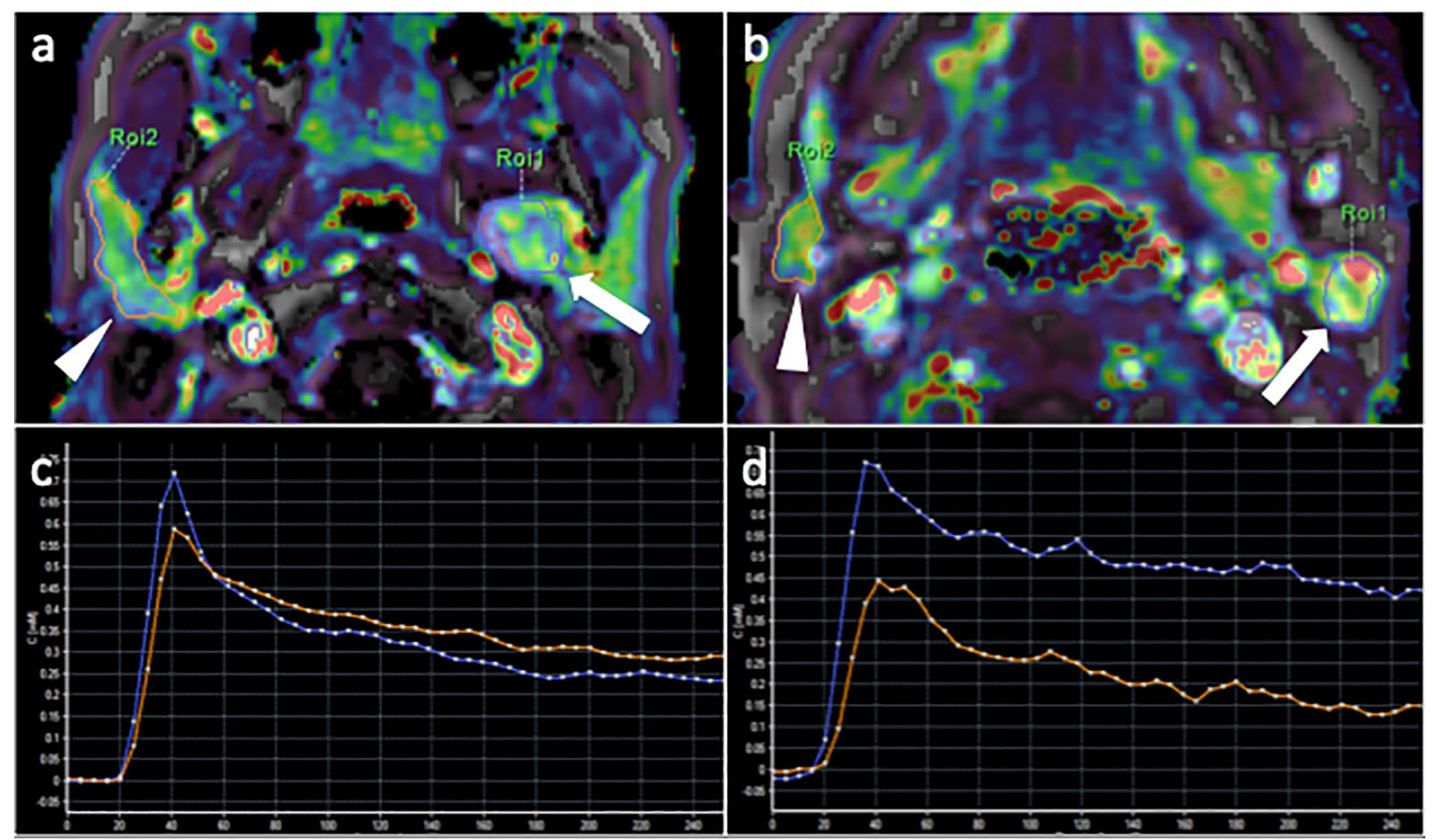

Fig. 4 68-year-old man and 70-year-old woman, respectively, with basal cell adenoma (a, c) and basal cell adenocarcinoma (b, d) of the left parotid gland. Comparison of derived DCE-MRI data. In a and b are represented color-coded maps of the area of all times enhancement curve $(A E C)$ with corresponding ROIs of the lesion (white arrows, blue line) and normal contralateral parenchyma (white arrowheads, orange line). In $\mathbf{c}$ and $\mathbf{d}$ are displayed the time-signal intensity

a reduction in the microvessel density) and, consequently, pharmacokinetic measures. In such cases, we generally observe a decrease in values of Ktrans, $V p$, and $A E C$, so benign tumors may show L/P Ktrans and $A E C$ greater than 1.0 , leading to false positive for malignancy.

Most of the literature studies investigating the potential of perfusion and diffusion MR imaging in the characterization of salivary gland tumors [10-15] described different imaging post-processing methods that are often not readily available for clinical routine. On the other hand, software applications using extended Tofts model [19] for permeability analysis of MR images nowadays are quite popular and generally easy to handle. They also are usually capable of generating "fancy" pixel-by-pixel color maps representing quantitative parameters whose variability, when taken for their absolute value, may lead to inconclusive results. Our study, through the introduction of the ratio between the tumor and the normal parenchyma, provided a curves of drawn ROIs (blue line representing lesion and orange line representing parenchyma). Morphology of tumor enhancement curve is similar in the two cases (early wash-in followed by washout); however, the malignant tumor curve sits well above the standard parenchyma curve at all times. In these cases, the calculated lesion/parenchyma ratios of $A E C$ were 0.868 for the adenoma and 3.392 for the adenocarcinoma

clinically feasible method that appeared to bypass the variability issues of the measures, present indications on how to use these color maps effectively, and introduce potential biomarkers easy to estimate and understand.

\section{Conclusion}

In conclusion, our study proved how the evaluation of pixelby-pixel DCE-MR parametric maps could be useful for recognizing the principal types of salivary gland tumors by the comparison between the lesion and the normal parenchyma. Moreover, the lesion/parenchyma ratio of the area of all times enhancement curve seems a very valuable biomarker for differentiating benign from malignant tumors. In our opinion, this type of analysis should always be included in the setting of a multiparametric imaging approach for these tumors. 

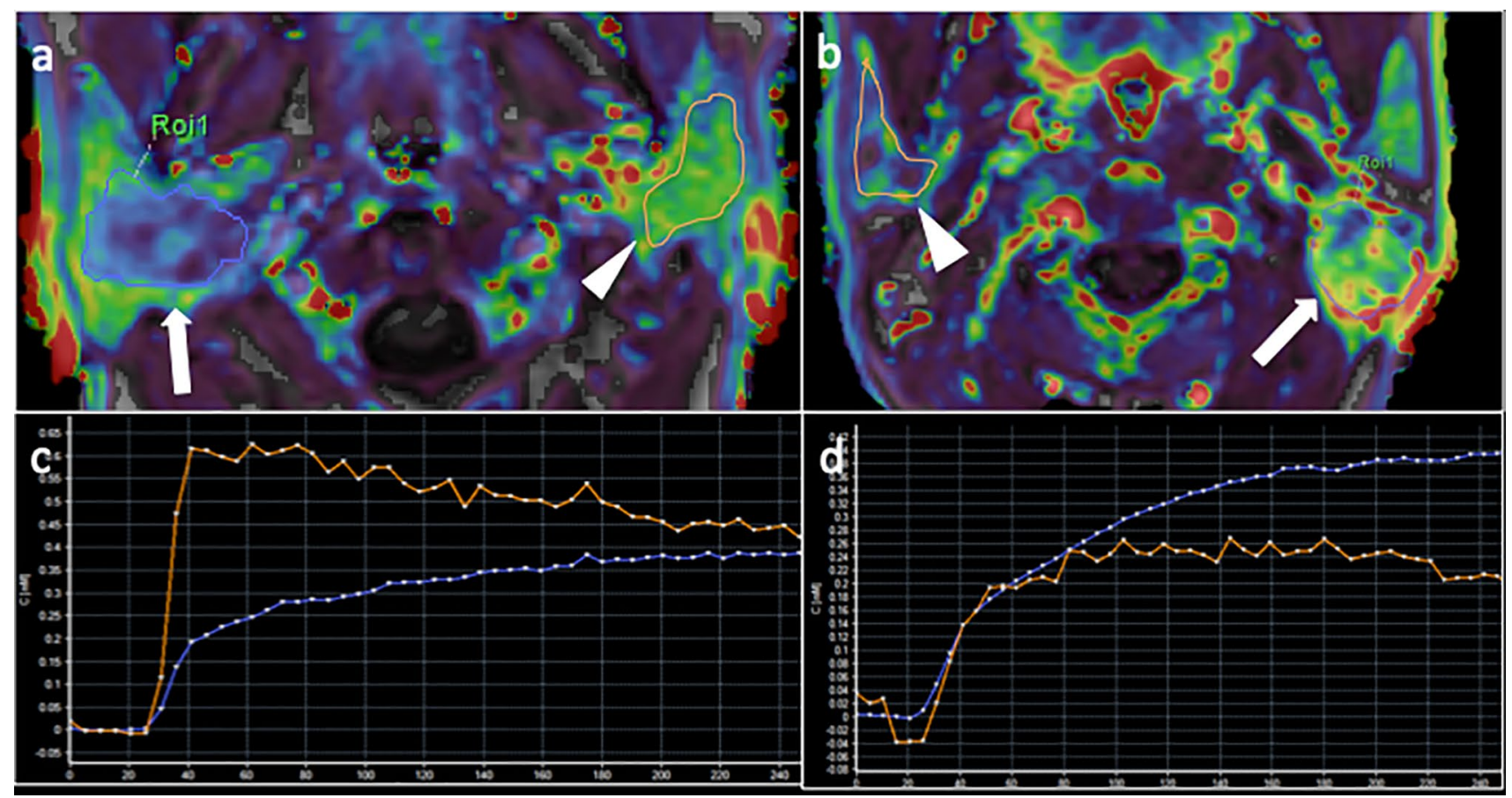

Fig. 5 70-year-old and 75-year-old men, respectively, with myoepithelioma of the right parotid gland (a, c) and squamous cell carcinoma (b, d) of the left parotid gland. Comparison of derived DCEMRI data. In $\mathbf{a}$ and $\mathbf{b}$ are represented color-coded maps of the area of all times enhancement curve $(A E C)$ with corresponding ROIs of the lesion (white arrows, blue line) and normal contralateral parenchyma (white arrowheads, orange line). In $\mathbf{c}$ and $\mathbf{d}$ are displayed the time-signal intensity curves of drawn ROIs (blue line represent- ing lesion and orange line representing parenchyma). Morphology of tumor enhancement curve is again similar in the two cases (early wash-in followed by a slower increase of enhancement); however, the benign tumor curve sits well under the standard parenchyma curve at all times. In these cases, calculated lesion/parenchyma ratios of $A E C$ were 0.622 for the myoepithelioma and 1.212 for the squamous cell carcinoma
Authors' contribution FM and LB are involved in conceptualization; FM, LNM, and VB helped in methodology; GBV, VB, EB, MP, and $\mathrm{CN}$ contributed to formal analysis and investigation; FM and GBV were involved in writing - original draft preparation; all authors helped in writing - review and editing; VM helped in supervision.

\section{Funding None.}

Availability of data and material yes.

\section{Declarations}

Conflict of interest All authors have no disclosures or conflicts of interest.

Ethics approval Institutional Board Review approved this study (protocol number: 2042/2019).

Consent to participate Informed consent was obtained from all patients included in the study.
Consent for publication Patients signed informed consent regarding publishing their data in anonymous form.

\section{References}

1. Sood S, McGurk M, Vaz F (2016) Management of salivary gland tumours: United Kingdom National Multidisciplinary Guidelines. J Laryngol Otol 130:S142-S149. https://doi.org/10.1017/s0022 215116000566

2. Abdel Razek AAK, Mukherji SK (2018) State-of-the-art imaging of salivary gland tumors. Neuroimaging Clin N Am 28:303-317. https://doi.org/10.1016/j.nic.2018.01.009

3. Dai YL, King AD (2018) State of the art MRI in head and neck cancer. Clin Radiol 73:45-59. https://doi.org/10.1016/j.crad.2017. 05.020

4. Prasad RS (2016) Parotid gland imaging. Otolaryngol Clin North Am 49:285-312. https://doi.org/10.1016/j.otc.2015.10.003

5. Abdel Razek AAK, Mukherji SK (2018) Imaging of minor salivary glands. Neuroimaging Clin N Am 28:295-302. https://doi. org/10.1016/j.nic.2018.01.008 
6. Vogl TJ, Albrecht MH, Nour-Eldin NA et al (2018) Assessment of salivary gland tumors using MRI and CT: impact of experience on diagnostic accuracy. Radiol Med 123(2):105-116. https://doi. org/10.1007/s11547-017-0813-z

7. Consamus EN, Smith D, Pina Oviedo S, Mody DR, Takei H (2015) Diagnostic accuracy of fine-needle aspiration cytology of salivary gland lesions: a 6-year retrospective review. J Am Soc Cytopathol 4:63-73. https://doi.org/10.1016/j.jasc.2014.11.003

8. Kim HJ, Kim JS (2018) Ultrasound-guided core needle biopsy in salivary glands: a meta-analysis. Laryngoscope 128:118-125. https://doi.org/10.1002/lary.26764

9. Haldar S, Sinnott JD, Tekeli KM, Turner SS, Howlett DC (2016) Biopsy of parotid masses: review of current techniques. World J Radiol 8:501-505. https://doi.org/10.4329/wjr.v8.i5.501

10. Sumi M, Nakamura T (2014) Head and neck tumours: combined MRI assessment based on IVIM and TIC analyses for the differentiation of tumors of different histological types. Eur Radiol 24:223-231. https://doi.org/10.1007/s00330-013-3002-z

11. Yamamoto T, Kimura H, Hayashi K, Imamura Y, Mori M (2018) Pseudo-continuous arterial spin labeling MR images in Warthin tumors and pleomorphic adenomas of the parotid gland: qualitative and quantitative analyses and their correlation with histopathologic and DWI and dynamic contrast enhanced MRI findings. Neuroradiology 60:803-812. https://doi.org/10.1007/ s00234-018-2046-9

12. Katayama I, Eida S, Fujita S, Hotokezaka Y, Sumi M, Nakamura $T$ (2017) Perfusion MR imaging detection of carcinoma arising from preexisting salivary gland pleomorphic adenoma by computer-Assisted analysis of time-signal intensity maps. PLoS ONE 12:e0178002. https://doi.org/10.1371/journal.pone.0178002

13. Lam PD, Kuribayashi A, Imaizumi A, Sakamoto J, Sumi Y, Yoshino $\mathrm{N}$ et al (2015) Differentiating benign and malignant salivary gland tumours: diagnostic criteria and the accuracy of dynamic contrast-enhanced MRI with high temporal resolution. Br J Radiol. https://doi.org/10.1259/bjr.20140685

14. Pietragalla M, Nardi C, Bonasera L et al (2020) The role of diffusion-weighted and dynamic contrast enhancement perfusion-weighted imaging in the evaluation of salivary glands neoplasms. Radiol Med 125(9):851-863. https://doi.org/10.1007/ s11547-020-01182-2
15. Sumi M, Van Cauteren M, Sumi T, Obara M, Ichikawa Y, Nakamura $T$ (2012) Salivary gland tumors: use of intravoxel incoherent motion MR imaging for assessment of diffusion and perfusion for the differentiation of benign from malignant tumors. Radiology 263:770-777. https://doi.org/10.1148/radiol.12111248

16. Kim H (2018) Variability in quantitative DCE-MRI: sources and solutions. J Nat Sci 4:e484

17. Evelhoch JL (1999) Key factors in the acquisition of contrast kinetic data for oncology. J Magn Reson Imaging 10:254-259. https://doi.org/10.1002/(SICI)1522-2586(199909)10:3\%3c254:: AID-JMRI5\%3e3.0.CO;2-9

18. Profile: DCE MRI quantification v.1.0. (2014) Quantitative imaging biomarkers alliance (QIBA)-Radiological society of North America. Web site. https://qibawiki.rsna.org/index.php/Profiles. updated July 1, 2012. Accessed December

19. Tofts PS, Brix G, Buckley DL, Evelhoch JL, Henderson E, Knopp MV et al (1999) Estimating kinetic parameters from dynamic contrast-enhanced T1-weighted MRI of a diffusable tracer: standardized quantities and symbols. J Magn Reson Imaging 10:223-232. https://doi.org/10.1002/(SICI)1522-2586(199909)10:3\%3c223: AID-JMRI2\%3e3.0.CO;2-S

20. Cuenod CA, Balvay D (2013) Perfusion and vascular permeability: basic concepts and measurement in DCE-CT and DCE-MRI. Diagn Interv Imaging 94:1187-1204. https://doi.org/10.1016/j. diii.2013.10.010

21. Som PM, Brandwein-Gensler MS (2011) Anatomy and pathology of the salivary glands. In: Som PM, Curtin HD (eds) Head and neck imaging, 5th edn. Elsevier Mosby, St. Louis, Pa, pp 2449-2468

22. van Rijswijk CS, Geirnaerdt MJ, Hogendoorn PC et al (2004) Soft-tissue tumors: value of static and dynamic gadopentetate dimeglumine-enhanced MR imaging in prediction of malignancy. Radiology 233:493-502. https://doi.org/10.1148/radiol.23320 31110

Publisher's Note Springer Nature remains neutral with regard to jurisdictional claims in published maps and institutional affiliations. 